\title{
Geographical differences in survival of dogs with non-Hodgkin lymphoma treated with a CHOP based chemotherapy protocol
}

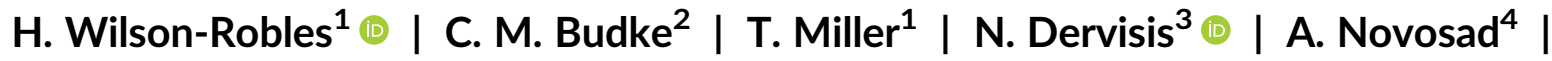 \\ Z. Wright ${ }^{5}$ | D. H. Thamm ${ }^{6} \mid$ K. Vickery ${ }^{7} \mid$ K. Burgess ${ }^{8} \mid$ M. Childress ${ }^{9} \mid$ J. Lori ${ }^{10}$ |

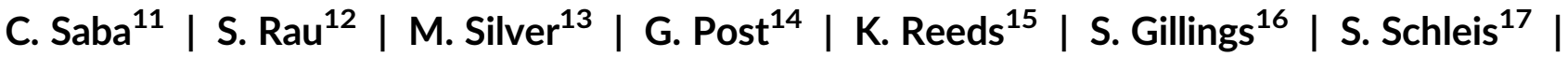 \\ T. Stein ${ }^{18} \mid$ B. Brugmann ${ }^{19} \mid$ C. DeRegis ${ }^{20} \mid$ O. Smrkovski ${ }^{21}$ | J. Lawrence ${ }^{11} \mid$ T. Laver ${ }^{22}$
}

${ }^{1}$ Small Animal Clinical Sciences Department, College of Veterinary Medicine, Texas A\&M University, College Station, Texas

${ }^{2}$ Veterinary Integrative Biosciences Department, College of Veterinary Medicine, Texas A\&M University, College Station, Texas

${ }^{3}$ Small Animal Clinical Sciences Department, Veterinary Medical Center, Michigan State University, East Lansing, Michigan

${ }^{4}$ Sugar Land Veterinary Specialists and Emergency Care, Sugar Land, Texas

${ }^{5}$ VCA Animal Diagnostic Clinic, Dallas, Texas

${ }^{6}$ Department of Clinical Sciences, College of Veterinary Medicine, Colorado State

University, Fort Collins, Colorado

${ }^{7}$ Hope Veterinary Specialists, Malvern,

Pennsylvania

${ }^{8}$ Department of Clinical Sciences, Cummings School of Veterinary Medicine, Tufts

University, North Grafton, Massachusetts

${ }^{9}$ Department of Veterinary Clinical Sciences, College of Veterinary Medicine, Purdue University, West Lafayette, Indiana

${ }^{10}$ Animal Emergency and Specialty Center, Parker, Colorado

${ }^{11}$ Department of Veterinary Clinical Sciences, College of Veterinary Medicine, University of Minnesota, St Paul, Minnesota

${ }^{12}$ Metropolitan Veterinary Associates, Norristown, Pennsylvania

${ }^{13} \mathrm{New}$ England Veterinary Oncology Group, Waltham, Massachusetts

${ }^{14}$ Department of Oncology, The Veterinary Cancer Center, Norwalk, Connecticut

${ }^{15}$ Veterinary Clinical Sciences Department, Center for Veterinary Health Sciences, Oklahoma State University, Stillwater, Oklahoma

${ }^{16}$ Summit Veterinary Referral Center, Tacoma, Washington
Background: In humans geographical differences in the incidence and presentation of various cancers have been reported. However, much of this information has not been collected in veterinary oncology.

Aims: The purpose of this study was to determine if a geographic difference in progression free survival exists for dogs with lymphoma treated within the US.

Materials and Methods: Medical records of 775 cases of canine lymphoma from 3 US regions (west, south and north), treated with CHOP chemotherapy, were retrospectively evaluated. Cases were collected from referral institutions and were required to have received at least one doxorubicin treatment and have follow up information regarding time to progression.

Results: Significant differences in sex $(p=0.05)$, weight $(p=0.049)$, stage $(p<0.001)$, immunophenotype $(p=<0.001)$, and number of doxorubicin doses $(p=0.001)$ were seen between regions. Upon univariate analysis, progression free survival (PFS) differed by region ( $p=0.006$ ), stage $(p=0.009)$, sub-stage $(p=0.0005)$, and immunophenotype $(p=0.001)$. A multivariable Cox regression model showed that dogs in the western region had a significantly shorter PFS when compared to the south and east.

Conclusions: PFS was significantly affected by stage, sub-stage and phenotype.

\section{KEYWORDS}

canine, CHOP, geographical differences, location, lymphoma 
${ }^{17}$ Clinical Sciences Department, College of Veterinary Medicine, Auburn University, Auburn, Alabama

${ }^{18}$ Medical Sciences Department, School of Veterinary Medicine, University of WisconsinMadison, Madison, Wisconsin

${ }^{19}$ Veterinary Clinical Sciences Department, School of Veterinary Medicine, Louisiana State University, Baton Rouge, Louisiana

${ }^{20}$ Pieper Memorial Veterinary Center, Middletown, Connecticut

${ }^{21}$ Small Animal Clinical Sciences Department, College of Veterinary Medicine, University of Tennessee, Knoxville, Tennessee

${ }^{22}$ Department of Small Animal Medicine and Surgery, College of Veterinary Medicine, University of Georgia, Athens, Georgia

Correspondence

H. Wilson-Robles, Small Animal Clinical Sciences Department, College of Veterinary Medicine, Texas A\&M University, 400

Raymond Stotzer Parkway, College Station, TX 77845

Email: HWilson@cvm.tamu.edu

\section{1 | INTRODUCTION}

The incidence of non-Hodgkin lymphoma (NHL) in humans has long been known to be variable based on region. The more developed regions tend to have the highest incidence of NHL including North America, Western Europe and Australia. ${ }^{1,2}$ Some areas of Africa with a high incidence of human immunodeficiency virus (HIV) and acquired immune deficiency syndrome also have a high incidence of $\mathrm{NHL}^{2}$ Trends in survival differences can also be seen by geographical regions for various tumour types including $\mathrm{NHL}$. In 1 study, evaluating 5-year NHL survival percentages for 10 European Countries and a total of 11 diverse areas of Europe, Cracow and Estonia had the lowest 5-year survival rates at $36.4 \%$ and $38.8 \%$, respectively, whereas Geneva and Saarland had the highest 5-year survival rates at $61.9 \%$ and $60.6 \%$, respectively, in the same year. ${ }^{3}$

Differences in treatment outcomes based on geography for various other tumour types have been reported in the human literature. One study evaluating the clinical presentation and severity of intravascular lymphoma (IVL) in Eastern and Western populations determined that $44 \%$ of Japanese patients had hemophagocytosis associated with their IVL, while none of the Western patients had hemophagocytosis. ${ }^{4}$ A study evaluating the treatment outcomes of patients with colorectal cancer demonstrated a decrease in overall survival in northern Manitoba compared with other areas of the province. ${ }^{5}$ Another study evaluating the level of care of patients with rectal cancer in the United States found that geography played a role in the dose and schedule of adjuvant of neoadjuvant therapy a patient received. This in turn affected survival. The number of cases that each treatment centre evaluated per year also influenced the rate of adjuvant and neoadjuvant therapies instituted. ${ }^{6}$ Another study evaluating prostate cancer survival in New South Wales determined that men living in rural areas and socio-economically disadvantaged areas were significantly more likely to die of prostate cancer than those in urban and wealthy areas. ${ }^{7}$ These differences may represent differences in availability of advanced medical care, rate of early detection and diagnosis, rate of commonly used screening tests, socioeconomic factors and differences in tumour subtype distributions among other causes. ${ }^{3,5,7}$

Lymphoma is one of the most common malignancies diagnosed in pet dogs in the United States. ${ }^{8-10}$ Lymphoma represents a diverse group of diseases similar to those seen in humans, with those of the non-Hodgkin B-cell phenotype being most commonly diagnosed. ${ }^{8}$ The aetiology of lymphoma in companion animals is generally unknown and likely multifactorial; however, a genetic predisposition with certain breed associations certainly exists. ${ }^{8,9}$ In addition, there is evidence that environmental factors such as exposure to herbicides and other chemicals may modify breedassociated risk. ${ }^{11-13}$ Introduction of doxorubicin-based chemotherapy protocols, specifically CHOP (a 15-25-week induction protocol for lymphoma including an alternating schedule of cyclophosphamide, doxorubicin, vincristine and prednisone), in the 1990s lead to improved survival for canine patients with median survival times reportedly between 275 and 397 days. ${ }^{14-17}$ Negative prognostic factors, including, but not limited to, sub-stage, T-cell immunophenotype, advanced stage and large body weight can reduce this expected survival time significantly. ${ }^{8,9,18-22}$ Anecdotally, many clinicians have reported that dogs treated with standard of care chemotherapy regimens in the Southern and Southeastern United States do not achieve the same survival or remission durations reported in the literature, regardless of negative prognostic factors. The aim of this study was to determine if a possible geographic difference exists in the first remission durations of pet dogs diagnosed and treated at referral institutions with doxorubicin-based chemotherapy in the continental United States. 


\section{2 | MATERIALS AND METHODS}

Medical records from 22 veterinary referral sites (academic and private practice) across the United States from 2004 to 2013 were examined retrospectively for dogs meeting the following inclusion criteria: a confirmed cytologic or histopathologic diagnosis of multicentric lymphoma (stage II-V) that was treated with a $\mathrm{CHOP}$ (Cyclophosphamide, hydroxydaunarubicin (doxorubicin), Oncovin (vincristine), prednisone) based protocol and for whom progressionfree survival (PFS) information was available. Each site identified eligible cases using their specific medical records systems and only included information for those dogs meeting the requirements above in the final Excel datasheet. Data collected included age, sex, breed, weight, state of residence, phenotype (T-cell, B-cell, other or unknown), stage (I-IV), sub-stage (a-asymptomatic or b-symptomatic), diagnostic/staging test results, PFS, chemotherapy protocol and number of doxorubicin doses. In addition, information regarding which staging tests were performed, that is, chest radiographs, bone marrow aspirate, abdominal ultrasound, flow cytometry, polymerase chain reaction for antigen receptor rearrangement and minimum database were also collected. Medical records were abstracted and recorded in an Excel (Microsoft, Redmond, Washington) spreadsheet provided by Heather Wilson-Robles. Centres were invited to participate through an invitation on the veterinary oncology list service (ACVIM360), through a call on the ACVIM webpage (acvim.org) or through a call at the Veterinary Cancer Society Meeting in October 2012. Any centres with eligible cases recorded on the Excel spreadsheet were included in this study.

\section{1 | Statistical analysis}

Geographic locations for the included dogs were divided into western United States (Colorado, Oregon and Washington), southern United States (Alabama, Arkansas, Florida, Georgia, Kansas, Kentucky, Louisiana, North Carolina, Oklahoma, South Carolina, Texas, Tennessee and Virginia) and northern United States (Connecticut, Illinois, Indiana, lowa, Massachusetts, Michigan, New Hampshire, New Jersey, New York, Pennsylvania, Rhode Island and Wisconsin) (Figure 1). Pearson's $\chi^{2}$ test was used to evaluate differences between categorical variables (sex, weight, stage, sub-stage, immunophenotype, doxorubicin doses and protocol number) by region. For analysis purposes, weight was dichotomized into those dogs weighing less than $15 \mathrm{~kg}$ and those dogs weighing $15 \mathrm{~kg}$ or more. The variable age was assessed for normality using the Shapiro-Wilk test. Since, the null hypothesis that the data came from a normally distributed population was rejected, the Kruskal-Wallis test was used to evaluate differences in age by region. Dogs with missing information on a specific variable were excluded from assessment of that variable.

The PFS was the primary endpoint of interest. For the purposes of this study, PFS is defined as the time point when a dog developed recurrence (enlarged lymph nodes with cytologic confirmation of lymphoma) after being in a complete clinical remission or when progressive disease was noted via objective tumour measurements. Relapse or disease progression was defined according to the response evaluation criteria for peripheral nodal lymphoma in dogs (v1.0). ${ }^{23}$ For each

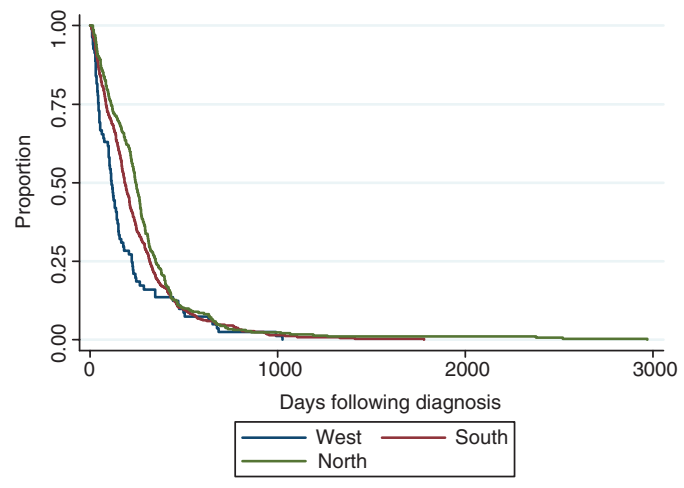

FIGURE 1 A Kaplan-Meier survival curve demonstrating the differences in progression-free survival between the 3 regions ( $\mathrm{n}=775)(P=.006)$. (Individual comparisons: north vs south $[P=.0329]$, north vs west $[P=.0002]$ and south vs west $[P=.0107]$, with statistical significance set at $[P<.0167]$.)

patient, this was defined as the interval between date of diagnosis and the date of relapse. The Kaplan-Meier method was used to obtain univariate descriptive statistics for time to first progression (the number of days which passed between diagnosis and recurrence or progressive disease) for the enrolled cases by geographic region, stage, sub-stage, immunophenotype, sex and weight. The log-rank test for equality across strata was used to evaluate survival estimates for the groups. For comparisons between more than 2 groups, individual comparisons were also made using the log-rank test with significance evaluated after applying a Bonferonni correction for multiple comparisons. A Cox proportional hazards model, with a single continuous predictor, was used to assess time to first progression by age. The Wald $\chi^{2}$ test was used to evaluate the association of this continuous variable [ie age] with PFS.

A multivariable Cox regression model was built to assess the association or effect of the potential predictor variables. Those variables with a $P$-value of .25 or less on univariate analysis were initially put into the multivariable model. Predictors were manually dropped from the model using a backward elimination process based on individual variable $P$-values. All possible 2 -way interactions between these variables were assessed. Collinearity was not specifically evaluated, but was biologically considered unlikely. The assumption of proportionality was confirmed by including time-dependent covariates in the model. Model fit was evaluated using Cox-Snell residuals. Analyses were performed using STATA software, Intercooled version 12.0. Statistical significance was assessed at $P<.05$.

\section{3 | RESULTS}

In total, 803 abstracted records were received and a total 775 dogs included in the analysis. Reasons for case exclusion included an intent to treat with CHOP chemotherapy but doxorubicin was never administered (1 case) and unconfirmed cytologic diagnoses (27 cases). Breeds represented included mixed breed ( $n=161,20.7 \%$ ), golden retriever $(n=97,12.5 \%$ ), Labrador retriever $(n=57,7.3 \%)$, boxer ( $n=28,3.6 \%$ ), German shepherd $(n=28,3.6 \%)$, rottweiler $(n=25$, $3.2 \%$ ), beagle $(n=22,2.8 \%)$, border collie $(n=24,3.1 \%)$, basset 
hound ( $n=17,2.2 \%$ ), miniature schnauzer ( $n=17,2.2 \%$ ), cocker spaniel ( $n=15,1.9 \%$ ), Jack Russell terrier $(n=12,1.5 \%)$, Shetland sheepdog ( $n=12,1.5 \%$ ), shih tzu ( $n=12,1.5 \%$ ), American Staffordshire terrier ( $n=11,1.4 \%)$, Australian shepherd $(n=10,1.3 \%)$, doberman pinscher ( $n=10,1.3 \%$ ), English bulldog ( $n=10,1.3 \%$ ), Scottish terrier $(n=10,1.3 \%)$, Welsh corgi $(n=10,1.3 \%)$ and other breeds with less than 10 representatives ( $n=193,24.9 \%$ ). A total of 81 dogs were included from the western region, 401 dogs were included from the southern region, and 293 dogs were included from the northern region. Study dogs comprised of 354 (45.7\%) neutered males, 302 (38.9\%) spayed females, 75 (9.7\%) intact males and 44 (5.7\%) intact females, with a median age at diagnosis of 8.0 years (range: 1.7-18.3 years). Of the 775 cases included in the study, 176 (22.7\%) cases had a histopathologic diagnosis of lymphoma, 428 cases had a cytologic diagnosis of lymphoma (55.2\%) and the method of diagnosis was not reported for 171 cases (22.1\%).

Sex $(P=.05)$, weight $(P=.049)$, stage $(P<.001)$, immunophenotype $(P=<.001)$ and number of doxorubicin doses $(P=.001)$ differed by region (Table 1$)$. Sex did not differ by region $(P=.583)$. The median number of doxorubicin doses administered for all dogs was 4.0 (range: 1-9) doses. In the west, dogs received a median of 3.0 (range: 1-8) doses of doxorubicin, in the south dogs received a median of 4.0 (range: 1-9) doses of doxorubicin and in the north dogs received a median of 4.0 (range: $1-9)$ doses of doxorubicin $(P=.001$ ).

TABLE 1 Patient demographics by region

\begin{tabular}{|c|c|c|c|c|}
\hline \multirow[b]{2}{*}{ Variable } & \multicolumn{3}{|c|}{ Number of cases by region ${ }^{1}(\%)$} & \multirow[b]{2}{*}{$P$-value } \\
\hline & West & South & North & \\
\hline \multicolumn{5}{|l|}{$\operatorname{Sex}(n=293)$} \\
\hline $\begin{array}{c}\text { Neutered } \\
\text { males }\end{array}$ & 37 (45.7\%) & $184(45.9 \%)$ & $133(45.4 \%)$ & .050 \\
\hline Intact males & $5(6.2 \%)$ & $34(8.5 \%)$ & $36(12.3 \%)$ & \\
\hline $\begin{array}{l}\text { Spayed } \\
\text { females }\end{array}$ & 37 (45.7\%) & $165(41.1 \%)$ & 100 (34.1\%) & \\
\hline $\begin{array}{l}\text { Intact } \\
\text { females }\end{array}$ & $2(2.5 \%)$ & $18(4.5 \%)$ & $24(8.2 \%)$ & \\
\hline \multicolumn{5}{|c|}{ Weight $(n=747)$} \\
\hline$<15$ kg & 32 (39.5\%) & $215(53.7 \%)$ & $129(48.5 \%)$ & .049 \\
\hline$\geq 15 \mathrm{~kg}$ & 49 (60.5\%) & 185 (46.3\%) & $137(51.5 \%)$ & \\
\hline \multicolumn{5}{|l|}{ Stage $(n=729)$} \\
\hline 1 & 0 & $1(0.3 \%)$ & 0 & $<.001$ \\
\hline II & $3(3.7 \%)$ & $8(2.1 \%)$ & $2(0.7 \%)$ & \\
\hline III & $54(66.7 \%)$ & $111(29.1 \%)$ & 85 (31.8\%) & \\
\hline IV & 14 (17.3\%) & $141(37.0 \%)$ & 97 (36.3\%) & \\
\hline V & $10(12.3 \%)$ & $120(31.5 \%)$ & $83(31.1 \%)$ & \\
\hline \multicolumn{5}{|c|}{ Sub-stage $(n=720)$} \\
\hline a & 59 (72.8\%) & $256(67.9 \%)$ & $164(62.6 \%)$ & .166 \\
\hline$b$ & $22(27.2 \%)$ & 121 (32.1\%) & 98 (37.4\%) & \\
\hline \multicolumn{5}{|c|}{ Immunophenotype $(n=775)$} \\
\hline T-cell & 10 (12.3\%) & $33(8.2 \%)$ & 46 (15.7\%) & $<.001$ \\
\hline B-cell & 17 (21.0\%) & $150(37.4 \%)$ & 169 (57.7\%) & \\
\hline Other & $1(1.2 \%)$ & 27 (6.7\%) & $12(4.1 \%)$ & \\
\hline Unknown & $53(65.4 \%)$ & $191(47.6 \%)$ & $66(22.5 \%)$ & \\
\hline
\end{tabular}

$P$-values in bold are statistically significant.
All included dogs experienced recurrence or progressive disease, with date of death also available for 598 dogs. The median PFS for all dogs enrolled was 204 days (interquartile range: 89-322 days). Upon univariate analysis, PFS differed significantly between geographic regions $(P=.006)$, stage $(P=.0094)$, sub-stage $(P<.001)$ and immunophenotype $(P<.001)$ (Table 2 , Figures $1-4)$. There was no difference in PFS by sex $(P=.921)$, weight $(P=.504)$ or age $(P=.121)$. Region, stage, sub-stage and immunophenotype were included in the final model (Table 3). The proportional hazards assumption was met. No interaction terms were significant. Dogs in the western region had a significantly shorter PFS when compared with the south (Hazard ratio, $H R=0.722)$ and east $(H R=0.623)$. Survival was also significantly affected by stage, sub-stage and phenotype (Table 3 ).

\section{4 | DISCUSSION}

The heterogeneous nature of $\mathrm{NHL}$ in dogs can make comparisons of survival and response to therapy difficult, especially in light of the fact that few dogs truly have histopathologic classification of their tumours. This is the first large descriptive study evaluating regional difference in the United States and serves to inform future prospective studies. The data gathered from this study are limited by the fact that only referral hospitals contributed cases, there were relatively few referral hospitals included in any given area and the numbers of dogs included are relatively low given the estimated canine populations for those areas. Given data provided in this study, geographic differences in NHL type and outcome are likely but underlying reasons for this have yet to be determined. Though full staging has become more commonplace, there were still a reasonable percentage of dogs in this group who were not fully staged or who did not have immunophenotyping performed.

In this study, a significant difference in median PFS was found between dogs residing in the northern region of the United States compared with those living in the southern and western states. Based on the prognostic factors that were available for evaluation in this study, no obvious reason exists to explain this difference. For example, the southern region had a lower percentage of T-cell lymphomas compared with the other 2 regions but did not have the longest PFS. The western region had the lowest percentage of stage $\mathrm{V}$ lymphomas (12\%), and yet the shortest median PFI when compared with the other 2 regions which each had $31 \%$ of their cases as stage $V$ lymphoma, and the northern region had the highest percentage of substage $b$ cases within its group yet had the longest median PFS. While results may be conflicting, it highlights the need to have strict classification criteria, staging tests and collection of all data points available for each case to avoid statistical error. For example, in the west, it is possible that stage migration (ie dogs may have been falsely put into a lower stage because a bone marrow aspirate was not performed) would occur with full staging, thus increasing the percentage of true stage $\vee$ dogs.

As in human studies, socio-economic differences may play a role in survival outcomes for our canine patients as well. Based on data from the US Census Bureau, the median household income by state was lowest in the South Eastern areas of the country with the 8 of 
TABLE 2 Univariable analysis for factors associated with progression-free survival (PFS)

\begin{tabular}{|c|c|c|}
\hline Variable & $\begin{array}{l}\text { Median and interquartile } \\
\text { range for PFS in days }\end{array}$ & $P$-value \\
\hline \multicolumn{3}{|l|}{ Region $(n=775)$} \\
\hline West $(n=81)$ & $114(45-222)$ & .006 \\
\hline South $(n=401)$ & $186(85-316)$ & \\
\hline North $(n=293)$ & $244(112-348)$ & \\
\hline \multicolumn{3}{|l|}{ Sex $(n=775)$} \\
\hline Intact males $(n=75)$ & $210(79-331)$ & \\
\hline Spayed females $(n=302)$ & $208(112-337)$ & \\
\hline Intact females $(n=44)$ & $191(136-346)$ & \\
\hline \multicolumn{3}{|l|}{ Weight $(n=747)$} \\
\hline$<15 \mathrm{~kg}(\mathrm{n}=376)$ & $205(95-329)$ & .504 \\
\hline \multicolumn{3}{|l|}{ Stage $(n=729)$} \\
\hline$I I(n=13)$ & $371(78-470)$ & \\
\hline III $(n=250)$ & $186(97-313)$ & \\
\hline $\mathrm{IV}(\mathrm{n}=252)$ & $214(95-333)$ & \\
\hline$V(n=213)$ & $180(75-315)$ & \\
\hline \multicolumn{3}{|l|}{ Sub-stage $(n=720)$} \\
\hline$a(n=479)$ & $215(101-343)$ & .0005 \\
\hline$b(n=241)$ & $159(71-277)$ & \\
\hline \multicolumn{3}{|l|}{ Immunophenotype $(\mathrm{n}=775)$} \\
\hline T-cell $(n=89)$ & $120(61-227)$ & .0011 \\
\hline
\end{tabular}

the 10 lowest median household incomes reported in southern states. ${ }^{24}$ Though all dogs included in this study were prescribed the same multidrug chemotherapy protocol, there may be differences in

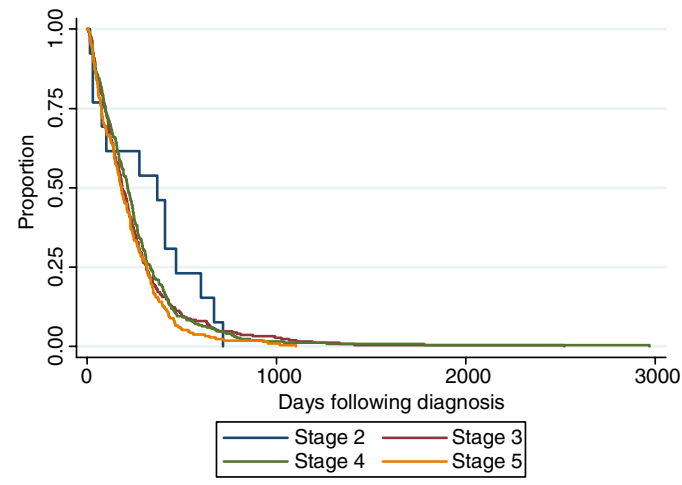

FIGURE 2 A Kaplan-Meier survival curve demonstrating the differences in progression-free survival (PFS) between the stages ( $\mathrm{n}=728)(P=.1395)$. Individual comparisons: stage 2 vs stage $3(P=.2559)$, stage 2 vs stage $4(P=.2633)$, stage 2 vs stage $5(P=.0739)$, stage 3 vs stage $4(P=.6358)$, stage 3 vs stage $5(P=.1556)$, stage 4 vs stage $5(P=.0649)$, with statistical significance set at $(P<.0083)$. Stage 1 was not assessed since only a single patient was in this category. time to diagnosis and protocol compliance in areas with lower household incomes. In addition, clinicians in each region may have variable criteria by which to institute starting dosages, dose reductions and chemotherapy delays, thus contributing to alterations in chemotherapy dose intensity. In humans, rural populations have been shown to be at increased risk of death because of cancer based on delayed

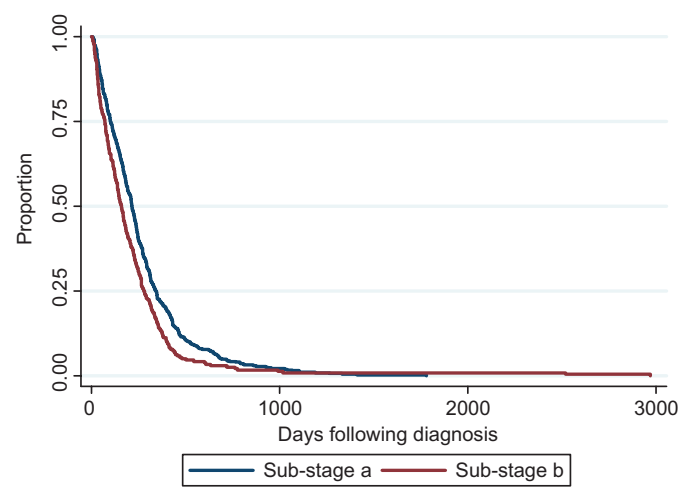

FIGURE 3 A Kaplan-Meier survival curve demonstrating a difference in progression-free survival (PFS) for dogs with sub-stage a (asymptomatic) and sub-stage b (symptomatic) disease $(n=720)$ $(P=.0005)$. 


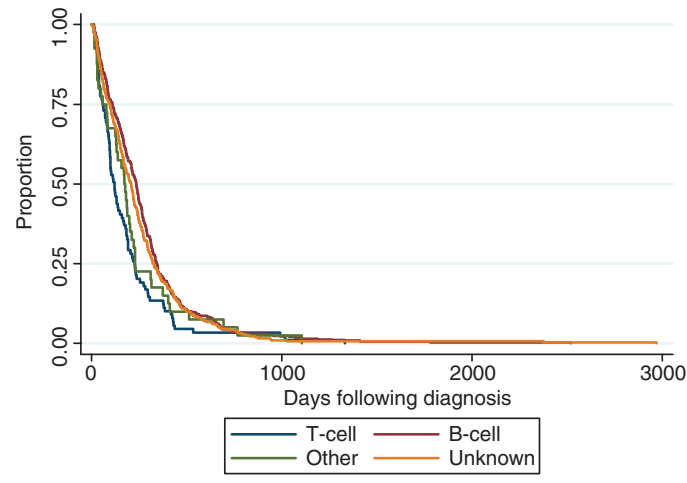

FIGURE 4 A Kaplan-Meier survival curve demonstrating a difference in progression-free survival (PFS) based on the immunophenotype ( $\mathrm{n}=465)(P=.0011)$. Individual comparisons: $T$-cell vs B-cell $(P=.0001)$, T-cell vs other $(P=.4044)$, T-cell vs unknown ( $P=.0028)$, B-cell vs other $(P=.1155)$, B-cell vs unknown $(P=.2694)$, other vs unknown $(P=.3537)$, with statistical significance set at $(P<.0083)$.

detection and limited treatment options. However, based on 2010 study produced by the Center for Business and Economic Research at the University of Alabama, ${ }^{25}$ the states with the highest percentage of rural populations were not well represented in this study. Five of the same states listed in the lowest income column are also in the most rural column, providing some overlap, especially in the Southern states; however, only $16.5 \%$ of the cases came from the lowest income states and only $4.7 \%$ of the cases came from the most rural states (Table 4).

In humans, viral infections with human T lymphotropic virus type 1 or chronic Epstein-Barr viral infections have been associated rarely with the development of lymphoid malignancies. A similar aetiology has been suggested in dogs with the study by Milman et al, ${ }^{26}$ where $64 \%$ of US dogs with lymphoma tested positive for the Epstein-Barr virus in serum. Though, no true link exists between a viral infection and the development of lymphoma in dogs, it is possible that an infectious aetiology could play a role in the geography, incidence and/or behaviour of lymphoma in dogs.

TABLE 3 Multivariable analysis for factors influencing progressionfree survival (PFS)

$\begin{array}{lllll} & & & & \begin{array}{l}\text { P- } \\ \text { Variable }\end{array} \\ \text { vegion } & \text { South vs west } & 0.722 & 0.560-0.930 & .012 \\ & \text { East vs west } & 0.623 & 0.476-0.814 & .001 \\ \text { Stage } & \text { II vs I } & 0.093 & 0.012-0.736 & .024 \\ & \text { III vs I } & 0.149 & 0.020-1.095 & .061 \\ & \text { IV vs I } & 0.152 & 0.021-1.117 & .064 \\ \text { Sub-stage } & \text { V vs I } & 0.167 & 0.228-1.218 & .077 \\ \text { Immunophenotype } & \text { b vs a } & 1.272 & 1.078-1.502 & .004 \\ & \text { B-cell vs T-cell } & 0.715 & 0.558-0.916 & .008 \\ & \text { Other vs T-cell } & 0.921 & 0.623-1.361 & .680 \\ & \text { Unknown vs T-cell } & 0.773 & 0.597-1.001 & .051\end{array}$

Abbreviations: $\mathrm{HR}$, hazard ratio; $\mathrm{Cl}$, confidence interval.

${ }^{1} \mathrm{HR}$ is presented for each sub-category (presented first) vs the baseline category (presented second).
Chronic antigenic stimulation has been associated with increased risk of NHL development in humans, especially in older individuals. ${ }^{27}$ One study found that respiratory and skin infections in humans were linked with an increased risk of $\mathrm{NHL}$, whereas gastrointestinal and urinary tract infections were not. ${ }^{28}$ Another study of 33 patients with rheumatoid arthritis demonstrated a shortened survival with lymphoma suggesting a more aggressive behaviour in cases with chronic inflammation. ${ }^{29}$ These patients were also more likely to develop diffuse large B-cell lymphoma, which carries a poorer prognosis other $\mathrm{NHL}$ subtypes. ${ }^{29,30}$ In veterinary medicine a study by Keller et $\mathrm{al}^{31}$ reported an increased risk of developing lymphoma in dogs with immune-mediated thrombocytopenia. Southern states are known for harbouring more infectious diseases because of a warmer climate, larger feral animal population and increased numbers of vectors (because of habitat requirements). A case report of an 11-year-old dog with concurrent T-cell lymphoma and Heterobilharzia suggested that, as in humans, schistosomiasis might contribute to the development of lymphoma. This aggressive lymphoma was refractory to treatment and the disease progressed rapidly. ${ }^{32}$ There is also an increase in the incidence of atopic conditions among pet populations in the South, again because of the warmer climates. In humans, B-cell lymphomas are thought to occasionally occur in individuals with chronic antigenic stimulation. ${ }^{27}$ The cellular signals from other cells in the inflammatory environment provide survival and proliferation signals that may lead to malignant transformation or sustained tumour cell growth. ${ }^{33,34}$ Though these causes more often are described in terms of incidence, they may also play a role in the biologic activity of the tumours they cause. HIV associated lymphomas (HAL) are known to have a very aggressive clinical behaviour compared with similar non-HIV related NHLs. This is thought to be related to an increased proliferation rate and a decrease in DNA aneuploidy in the HAL cases suggesting a difference in tumour pathogenesis. ${ }^{35}$ The same phenomenon is seen in cats with a positive retroviral status that develop lymphoid malignancies. ${ }^{36,37}$ It is beyond the scope of this study to evaluate these cases for possible co-morbidities at this time. Future studies will be needed to determine antigenic stimulation plays a role geographically in canine lymphoma.

In a review of the current literature, the regional PFS differences reported in this study appear to be consistent with other published reports. The median PFS for all animals in this study falls short of the 282 days reported by Garrett et al, ${ }^{14}$ it is similar to what has been reported more recently. ${ }^{38,39}$ The report from Garret et al was based on patients treated at the University of Wisconsin, which contributed 48 cases to this study. The median PFS for the Wisconsin-based cases in this study was determined to be 270 days, similar to what was reported back in 2002. Curran et al reported a median PFS of 176 days while Burton et al reported a median PFS of 140 days. These studies were performed on patients treated at Colorado State University. ${ }^{38}$ Interestingly, 44 cases in this study came from Colorado and the PFS for those cases was 142 days, half of what is seen in Wisconsin but similar to what has been previously reported. Dr. Sorenmo's study reported a median PFS of 219 days for the patients treated at the University of Pennsylvania, ${ }^{39}$ though in this study 63 dogs from the state of Pennsylvania were included and the median PFS for this group was 306 days, the longest of any state 
TABLE 4 Progression-free survival by state and region

\begin{tabular}{|lrrrrr|} 
State & Region & $\begin{array}{r}\text { Number } \\
\text { enrolled }\end{array}$ & $\begin{array}{c}\text { Min } \\
\text { (days) }\end{array}$ & $\begin{array}{c}\text { Median } \\
\text { (days) }\end{array}$ & $\begin{array}{c}\text { Max } \\
\text { (days) }\end{array}$ \\
\hline CO & West & 44 & 10 & 142 & 1027 \\
\hline OR & West & 1 & 18 & 18 & 18 \\
\hline WA & West & 36 & 8 & 49 & 475 \\
\hline AR & South & 1 & 290 & 290 & 290 \\
\hline KS & South & 49 & 18 & 260 & 876 \\
\hline LA & South & 8 & 28 & 47 & 161 \\
\hline OK & South & 20 & 34 & 164 & 418 \\
\hline TX & South & 105 & 4 & 173 & 1781 \\
\hline AL & South & 31 & 43 & 210 & 1104 \\
\hline FL & South & 6 & 7 & 165 & 408 \\
\hline GA & South & 44 & 6 & 207 & 954 \\
\hline KY & South & 5 & 14 & 151 & 926 \\
\hline NC & South & 69 & 7 & 188 & 1412 \\
\hline SC & South & 1 & 347 & 347 & 347 \\
\hline TN & South & 61 & 10 & 184 & 789 \\
\hline VA & South & 1 & 125 & 125 & 125 \\
\hline IA & North & 2 & 227 & 229 & 232 \\
\hline IL & North & 12 & 30 & 174 & 400 \\
\hline IN & North & 34 & 14 & 192 & 1068 \\
\hline MI & North & 35 & 22 & 167 & 2521 \\
\hline WI & North & 48 & 15 & 270 & 860 \\
\hline CT & North & 22 & 22 & 222 & 463 \\
\hline MA & North & 41 & 89 & 260 & 2971 \\
\hline NH & North & 3 & 186 & 203 & 234 \\
\hline NJ & North & 29 & 20 & 166 & 695 \\
\hline NY & North & 2 & 55 & 184 & 313 \\
\hline PA & North & 63 & 15 & 306 & 1266 \\
\hline RI & North & 2 & 239 & 251 & 263 \\
\hline & & & & \\
\hline
\end{tabular}

from which more than 1 dog was included. A study from Cornell University in 2010 determined that the PFS for their control group ( $L$ CHOP, 71 cases) was 298 days. ${ }^{40}$ Only 2 dogs from New York were included in this study (PFS 184 days); however, cases from surrounding states reported very similar PFS times (PA 63 cases, 306 days; MA 41 cases, 260 days; RI 2 cases, 251 days; CT 22 cases, 222 days) (Table 4).

In another recently published paper by Ruple et al, ${ }^{41}$ a difference in the geographic distribution of lymphoma subtypes in Golden retrievers was noted. In this study, dogs in the Northeast and East North Central regions were more likely to be diagnosed with T-zone lymphoma than B-cell lymphoma. Given the indolent nature of T-zone lymphoma in dogs, it would stand to reason that these dogs would likely live longer than some of other subtypes of lymphoma diagnosed in the dog. T-zone lymphoma cases were not included in this study, which focused on intermediate or high grade lymphomas treated with the CHOP protocol. However, this study does support the idea that geographic differences in canine lymphoma may exist and may be environmentally driven.

Limitations to the study include its retrospective nature, lack of standardized criteria or requirement for full staging, lack of histologic diagnoses contributing to NHL subtype, inclusion of only a referral population of dogs and inherent difficulties with chemotherapy protocols. It is unknown if a referral population of dogs with lymphoma is truly representative of the disease in these regions. Despite these limitations, the purpose of the study was simply to identify if geographic differences in NHL do exist across the United States. Indeed, preliminary results here support our hypothesis that there are geographic differences amongst dogs with NHL, although it is still unclear how multifactorial these differences are and how much influence prognostic factors may have on outcome. Further studies should strive to prospectively evaluate geographic differences through strict standardized means in order to better elucidate underlying mechanisms contributing to differences.

In conclusion, these data suggest that there may indeed be geographical differences in the PFS of dogs with lymphoma for reasons other than the traditionally considered negative prognostic factors. The authors of this article were unable to determine a cause for the differences in PFS between regions. Limitations of this study include the retrospective nature of the study, the collection of cases from referral hospitals only, the relatively small number of hospitals per region which submitted cases and the relatively low number of dogs considered given the estimated canine populations in those areas. These limitations certainly allow for the possibility of alternative explanations for this data. Regretfully, too few of the patients included in this study have histopathology confirming the specific type of lymphoma to investigate this further. Future studies will be needed to determine the underlying cause of these differences.

\section{REFERENCES}

1. Fisher SG, Fisher RI. The epidemiology of non-Hodgkin's lymphoma. Oncogene. 2004;23(38):6524-6534. doi:10.1038/sj.onc.1207843.

2. Roche H-L. 2014. Global Incidence of non-Hodgkin lymphoma, Globican. www.roche.com/backgrounder_epidemiology_of_cancer.pdf.

3. Gondos A, Bray F, Hakulinen T, Brenner H. Trends in cancer survival in 11 European populations from 1990 to 2009: a model-based analysis. Ann Oncol. 2009;20(3):564-573. doi:10.1093/annonc/mdn639.

4. Ferreri AJ, Dognini GP, Campo E, et al. Variations in clinical presentation, frequency of hemophagocytosis and clinical behavior of intravascular lymphoma diagnosed in different geographical regions. Haematologica. 2007;92(4):486-492.

5. Helewa RM, Turner D, Wirtzfeld D, et al. Does geography influence the treatment and outcomes of colorectal cancer? A population-based analysis. World J Surg Oncol. 2013;11:140. doi:10.1186/1477-781911-140.

6. Monson JR, Probst CP, Wexner SD, et al. Failure of evidence-based cancer care in the United States: the association between rectal cancer treatment, cancer center volume, and geography. Ann Surg. 2014;260(4):625-631; discussion 31-2. doi:10.1097/sla.000000000 0000928.

7. Yu XQ, Luo Q, Smith DP, O'Connell DL, Baade PD. Geographic variation in prostate cancer survival in New South Wales. Med J Aust. 2014;200(10):586-590.

8. Vail DM, Pinkerton ME, Young KM. Canine lymphoma and lymphoid leukemias. In: Withrow SJ, Vail D, Page RL, eds. Small Animal Clinical Oncology. 5th ed. St. Louis, MS: Elsevier Saunders; 2013:608-627.

9. Ettinger SN. Principles of treatment for canine lymphoma. Clin Tech Small Anim Pract. 2003;18(2):92-97. doi:10.1053/svms.2003.36622.

10. Teske E. Canine malignant lymphoma: a review and comparison with human non-Hodgkin's lymphoma. Vet Q. 1994;16(4):209-219. doi:10.1080/01652176.1994.9694451.

11. Hayes HM, Tarone RE, Cantor KP. On the association between canine malignant lymphoma and opportunity for exposure to 2,4- 
dichlorophenoxyacetic acid. Environ Res. 1995;70(2):119-125. doi:10. 1006/enrs.1995.1056.

12. Hayes HM, Tarone RE, Cantor KP, Jessen CR, McCurnin DM, Richardson RC. Case-control study of canine malignant lymphoma: positive association with dog owner's use of 2,4dichlorophenoxyacetic acid herbicides. I Natl Cancer Inst. 1991; 83(17):1226-1231.

13. Reynolds PM, Reif JS, Ramsdell HS, Tessari JD. Canine exposure to herbicide-treated lawns and urinary excretion of 2,4dichlorophenoxyacetic acid. Cancer Epidemiol Biomarkers Prev. 1994; 3(3):233-237.

14. Garrett LD, Thamm DH, Chun R, Dudley R, Vail DM. Evaluation of a 6-month chemotherapy protocol with no maintenance therapy for dogs with lymphoma. J Vet Intern Med. 2002;16(6):704-709.

15. MacDonald VS, Thamm DH, Kurzman ID, Turek MM, Vail DM. Does $\mathrm{L}$-asparaginase influence efficacy or toxicity when added to a standard CHOP protocol for dogs with lymphoma? J Vet Intern Med. 2005;19(5):732-736.

16. Chun R, Garrett LD, Vail DM. Evaluation of a high-dose chemotherapy protocol with no maintenance therapy for dogs with lymphoma. $J$ Vet Intern Med. 2000;14(2):120-124.

17. Hosoya K, Kisseberth WC, Lord LK, et al. Comparison of COAP and UW-19 protocols for dogs with multicentric lymphoma. J Vet Intern Med. 2007;21(6):1355-1363.

18. Kiupel M, Teske E, Bostock D. Prognostic factors for treated canine malignant lymphoma. Vet Pathol. 1999;36(4):292-300.

19. Teske $E$, van Heerde $P$, Rutteman GR, Kurzman ID, Moore PF, MacEwen EG. Prognostic factors for treatment of malignant lymphoma in dogs. J Am Vet Med Assoc. 1994;205(12):1722-1728.

20. Rosenberg MP, Matus RE, Patnaik AK. Prognostic factors in dogs with lymphoma and associated hypercalcemia. J Vet Intern Med. 1991;5(5):268-271.

21. Keller ET, MacEwen EG, Rosenthal RC, Helfand SC, Fox LE. Evaluation of prognostic factors and sequential combination chemotherapy with doxorubicin for canine lymphoma. J Vet Intern Med. 1993; 7(5):289-295.

22. Price GS, Page RL, Fischer BM, Levine JF, Gerig TM. Efficacy and toxicity of doxorubicin/cyclophosphamide maintenance therapy in dogs with multicentric lymphosarcoma. J Vet Intern Med. 1991;5(5):259-262.

23. Vail DM, Michels GM, Khanna C, Selting KA, London CA, Veterinary Cooperative Oncology Group. Response evaluation criteria for peripheral nodal lymphoma in dogs (v1.0) - a Veterinary Cooperative Oncology Group (VCOG) consensus document. Vet Comp Oncol. 2010;8(1):28-37. doi:10.1111/j.1476-5829.2009.00200.x.

24. Bureau UC. State and Country Quick Facts. 2/24/14. Report No. P60-252, http://www.census.gov/topics/income-poverty/income. html. Published by the United States Census.

25. Research BfBaE. Urban Rural by State 2010. The Center for Economic Research, The University of Alabama. http://cber.cba.ua.edu/2010.

26. Milman G, Smith KC, Erles K. Serological detection of Epstein-Barr virus infection in dogs and cats. Vet Microbiol. 2011;150(1-2):15-20. doi:10.1016/j.vetmic.2010.12.013.

27. Smedby KE, Baecklund E, Askling J. Malignant lymphomas in autoimmunity and inflammation: a review of risks, risk factors, and lymphoma characteristics. Cancer Epidemiol Biomarers Prev. 2006 15(11):2069-2077. doi:10.1158/1055-9965.Epi-06-0300.

28. Anderson LA, Atman AA, McShane CM, Titmarsh GJ, Engels EA, Koshiol J. Common infection-related conditions and risk of lymphoid malignancies in older individuals. Br J Cancer. 2014;110(11):27962803. doi:10.1038/bjc.2014.173.
29. Baecklund E, Sundstrom C, Ekbom A, et al. Lymphoma subtypes in patients with rheumatoid arthritis - Increased proportion of diffuse large B cell lymphoma. Arthritis Rheum. 2003;48(6):1543-1550. doi:10.1002/art.11144

30. Evans LS, Hancock BW. Non-Hodgkin lymphoma. Lancet. 2003;362(9378):139-146. doi:10.1016/S0140-6736(03)13868-8.

31. Keller ET. Immune-mediated disease as a risk factor for canine lymphoma. Cancer. 1992;70(9):2334-2337. doi:10.1002/1097-0142 (19921101)70:9<2334::Aid-Cncr2820700920>3.0.Co;2-7.

32. Stone RH, Frontera-Acevedo K, Saba CF, Ambrose D, Moorhead AR, Brown CA. Lymphosarcoma associated with Heterobilharzia americana infection in a dog. J Vet Diagn Invest. 2011;23(5):1065-1070. doi:10.1177/ 1040638711416972

33. Seifert M, Scholtysik R, Kuppers R. Origin and pathogenesis of B cell lymphomas. Methods Mol Biol. 2013;971:1-25. doi:10.1007/978-162703-269-8_1.

34. Zucca E, Bertoni F, Vannata B, Cavalli F. Emerging role of infectious etiologies in the pathogenesis of marginal zone B-cell lymphomas. Clin Cancer Res. 2014;20(20):5207-5216. doi:10.1158/1078-0432. ccr-14-0496.

35. McDunn SH, Winter JN, Variakojis D, et al. Human immunodeficiency virus-related lymphomas: a possible association between tumor proliferation, lack of ploidy anomalies, and immune deficiency. J Clin Oncol. 1991;9(8):1334-1340.

36. Vail DM, Moore AS, Ogilvie GK, Volk LM. Feline lymphoma (145 cases): proliferation indices, cluster of differentiation 3 immunoreactivity, and their association with prognosis in 90 cats. J Vet Intern Med. 1998;12(5):349-354.

37. Mooney SC, Hayes AA, MacEwen EG, Matus RE, Geary A, Shurgot BA. Treatment and prognostic factors in lymphoma in cats: 103 cases (1977-1981). J Am Vet Med Assoc. 1989;194(5):696-702.

38. Curran K, Thamm DH. Retrospective analysis for treatment of naive canine multicentric lymphoma with a 15-week, maintenance-free CHOP protocol. Vet Comp Oncol. 2016;14 (suppl 1):147-155. doi:10.1111/vco.12163.

39. Sorenmo K, Overley B, Krick E, Ferrara T, LaBlanc A, Shofer F. Outcome and toxicity associated with a dose-intensified, maintenancefree CHOP-based chemotherapy protocol in canine lymphoma: 130 cases. Vet Comp Oncol. 2010;8(3):196-208. doi:10.1111/j.14765829.2010.00222.x.

40. Rassnick KM, Bailey DB, Malone EK, et al. Comparison between $\mathrm{L}-\mathrm{CHOP}$ and an L-CHOP protocol with interposed treatments of CCNU and MOPP (L-CHOP-CCNU-MOPP) for lymphoma in dogs. Vet Comp Oncol. 2010;8(4):243-253. doi:10.1111/j.14765829.2010.00224.x.

41. Ruple A, Avery AC, Morley PS. Differences in the geographic distribution of lymphoma subtypes in Golden retrievers in the USA. Vet Comp Oncol. 2016. doi:10.1111/vco.12258.

How to cite this article: Wilson-Robles $\mathrm{H}$, Budke CM, Miller T, Dervisis N, Novosad A, Wright Z, Thamm DH, Vickery K, Burgess K, Childress M, Lori J, Saba C, Rau S, Silver M, Post G, ReedsK, Gillings S, Schleis S, Stein T, Brugmann B, DeRegis C, Smrkovski O, Lawrence, J, Laver T. Geographical differences in survival of dogs with non-Hodgkin lymphoma treated with a CHOP based chemotherapy protocol. Vet Comp Oncol. 2017. https://doi.org/10.1111/vco.12302. 